\title{
O perfil da populaçáo infantil com suspeita de diagnóstico de transtorno do espectro autista atendida por um Centro Especializado em Reabilitação de uma cidade do Sul do Brasil
}

\author{
I ${ }^{1}$ Carla Cecília Rocha, ${ }^{2}$ Sara Mariane Velasques de Souza, ${ }^{3}$ André Felipe Costa, \\ ${ }^{4}$ João Rodrigo Maciel Portes I
}

Resumo: O Centro Especializado em Reabilitaçấo (CERII) é um serviço do SUS que trabalha interdisciplinarmente na avaliaçáo e reabilitaçáo de pessoas com deficiência, incluindo a população com autismo. O objetivo desta pesquisa foi caracterizar o perfil da população infantil com suspeita de transtorno do espectro autista atendida por um CER-II do Sul do Brasil, levantar fontes, motivos de encaminhamentos, características sociodemográficas, instrumentos psicométricos utilizados nas avaliaçóes e os níveis de diagnóstico recebido. Neste estudo documental retrospectivo, descritivo e exploratório-quantitativo, foram analisados 685 prontuários de usuários com idade de zero a 12 anos, que fizeram avaliação entre 2014 até dezembro de 2017. Os resultados mostraram que a média de idade da amostra é de 5,47 anos, sendo 83,8\% do sexo masculino, $42 \%$ encontram-se na educação infantil e $59,7 \%$ fazem parte de uma família nuclear. Os sinais que mais motivaram encaminhamentos foram os déficits de linguagem $(58,8 \%)$ e comportamentos externalizantes (56,9\%); 30,1\% da amostra obtiveram diagnóstico de TEA confirmado e o instrumento mais utilizado foi o $\mathrm{ABC}(70,5 \%)$. Observase a efetividade da aplicaçáo do serviço proposto por essa política pública, destacando-se a necessidade de instrumentos qualificados para avaliação do diagnóstico e capacitação continuada para profissionais que nela atuam.

> Palavras-chave: autismo; crianças; SUS.

\author{
${ }^{1}$ Universidade do Vale do Itajaí. \\ Itajaí-SC, Brasil (caarla.rocha@ \\ hotmail.com). \\ ORCID: 0000-0002-2257-4334 \\ 2 Universidade do Vale do Itajaí. \\ Itajaí-SC, Brasil (sarahvelank@ \\ gmail.com). \\ ORCID: 0000-0002-0377-1463 \\ ${ }^{3}$ Universidade do Vale do Itajaí. \\ Itajaí-SC, Brasil \\ (andrefelipedacosta@edu.univali.br). \\ ORCID: 0000-0002-0975-9836 \\ ${ }^{4}$ Universidade do Vale do Itajaí. \\ Itajaí-SC, Brasil (joaorodrigo@ \\ univali.br). \\ ORCID: 0000-0003-2512-4491
}

Recebido em: 25/02/2019 Revisado em: 16/07/2019 Aprovado em: 05/08/2019 


\section{Introdução}

Desde meados dos anos 1950, é notável o aumento no número de leis e conferências relacionadas aos serviços de saúde para o atendimento às crianças com deficiência e transtornos mentais (MAZZOTTA, 1999). Leis como a Constituição de 1988 e o Estatuto da Criança do Adolescente de 1990, que garantem direitos e obrigaçóes quanto aos cuidados de saúde, exemplificam isso. Um marco na conquista desses direitos é a Convenção Internacional dos Direitos da Pessoa com Deficiência, que preconiza o respeito pela dignidade, autonomia e independência das pessoas com deficiência, e que tem em seus princípios a valorizaçáo da não discriminação e a inclusão na sociedade (BRASIL, 2009). Esse marco internacional inspirou a Lei Brasileira de Inclusão no 13.146 , que tem como objetivos o asseguramento e a promoção de condiçôes iguais para as pessoas com deficiência (BRASIL, 2015).

Atualmente, as políticas públicas garantem serviços de apoio especializado para a população com deficiência dentro do Sistema Único de Saúde (SUS). Uma dessas políticas é o Centro Especializado em Reabilitação (CER II), oriunda do Plano Viver Sem Limites, datado em 2011, mas instituída na Rede de Saúde de Cuidados à Pessoa com Deficiência, que visa promover autonomia e independência das pessoas com deficiência, além de garantir seus direitos (BRASIL, 2013; 2012a). Como parte da rede, os CERs podem atender pessoas com deficiência que possuem demandas de reabilitação física, auditiva, intelectual e/ou visual, trabalhando de forma interdisciplinar, variando com a demanda de cada CER (CAMPOS; SOUZA; MENDES, 2015). Segundo o Ministério da Saúde, em 2017 havia 187 CER habilitados (BRASIL, 2017).

Uma das populaçóes atendidas no CER II são crianças com transtorno do espectro autista (TEA). O TEA é caracterizado principalmente por prejuízos na sua comunicação social recíproca e na sua interação social, além de apresentar comportamentos padronizados, restritos e repetitivos. Os sintomas geralmente são identificados aos dois anos de idade, mesmo que alguns indícios mais evidentes possam ser observados nos primeiros 12 meses de idade. Muitos dos indivíduos com TEA também podem apresentar comprometimento intelectual e de linguagem (APA, 2014). Segundo a Política Nacional de Proteção dos Direitos da Pessoa com Transtorno do Espectro Autista, a Lei no 12.764 de 27 de novembro de 2012, pessoas com TEA são consideradas pessoas com deficiência (BRASIL, 2012b). 
Entretanto, ainda há um grande debate sobre as estratégias de cuidado para essa população. Alguns autores observam a existência de duas cartilhas organizadas pelo Ministério da Saúde, em 2013, para orientar o tratamento de pessoas com TEA no SUS. Esses dois documentos entram em conflito no modo como a política deverá ser executada. O primeiro, "Diretrizes de Atenção à Reabilitação da Pessoa com Transtorno do Espectro Autista (TEA)", observa o autismo no campo da deficiência e intervençôes mais focadas em reabilitação. Já o segundo, "Linha de Cuidado para a Atenção às Pessoas com Transtornos do Espectro do Autismo e suas Famílias na Rede de Atenção Psicossocial do Sistema Único de Saúde", remete o TEA ao campo dos transtornos mentais, observando que os cuidados sejam feitos juntos à rede de atenção psicossocial. Por fim, os autores concluem que há pontos convergentes em ambos os modelos, e que a finalidade da discussão é mais política do que clínica e epistemológica, cabendo ao Ministério da Saúde garantir a mediação desses modelos, para que a tensão por poder não tome o protagonismo na discussão (OLIVEIRA et al., 2017).

Apesar da escassez de dados epidemiológicos dos países em desenvolvimento, até o ano de 2016, o Brasil estava entre os poucos países que realizavam pesquisas nessa área. Uma das pesquisas era um estudo piloto sobre prevalência de TEA, com amostra de 1.470 crianças, entre sete a 12 anos, realizado em Atibaia, interior do estado de Sáo Paulo. Os dados obtidos foram de 0,3\% de prevalência em TEA (PAULA et al., 2011a; 2011b). O estudo de Portolese et al. (2017) nas regiôes metropolitanas de Goiânia, Fortaleza, Belo Horizonte e Manaus, contou com uma amostra de 1.715 estudantes, incluindo crianças e adolescentes de seis a 16 anos, e constatou a prevalência de $1 \%$ de TEA.

O parâmetro mais utilizado atualmente é do Center for Disease Control, que revelou uma prevalência de uma para 68 crianças com TEA, ou seja, uma taxa de 1,47\% (CHRISTENSEN et al., 2016). Quanto à populaçáo infantil com TEA no Brasil, estimava-se que na faixa etária de crianças pré-escolares de zero a quatro anos, de um total de 16.386 .239 crianças, 114.704 poderiam ser diagnosticadas com TEA (BOSA; TEIXEIRA, 2017).

Presmanes et al. (2014) apontam que a alta prevalência de diagnósticos de TEA pode estar relacionada com a maior sensibilidade dos instrumentos utilizados para diagnóstico, embora esses recursos possam ser insuficientes para diagnosticar as 
especificidades do TEA e assim produzir falsos positivos. Deve-se também levar em conta o aumento de serviços de referência que trabalham com a populaçáo que possui TEA, como CAPSi e CER, que contribuem para o aumento de diagnóstico dessa população (BRASIL, 2012). E ainda, a ampliação do conhecimento sobre o TEA entre profissionais da saúde que trabalham com essa população, educadores e a comunidade em geral (HILL; ZUCKERMAN; FOMBONNE, 2014).

Dessa forma, é visível a importância de pesquisas que abordam dados epidemiológicos sobre a população infantil com TEA no âmbito da saúde pública brasileira. Esses dados podem auxiliar na formulação de políticas públicas, assim como no maior conhecimento acerca da populaçáo atendida e principais demandas atingidas naquela região para maior qualificação do atendimento. A produção de pesquisas sobre TEA em Centros Especializados - como o CER -contribui para o saber social e científico em constante desenvolvimento, além de auxiliar na efetivação da política pública proporcionada ao público-alvo. Além disso, há carência de dados epidemiológicos no Brasil relacionados a essa população, sobretudo em serviços do SUS, como o CER.

Dessa maneira, o presente trabalho visa caracterizar o perfil da população infantil com suspeita de diagnóstico de transtorno do espectro autista atendida por um CER II no sul do Brasil, objetivando levantar as fontes e motivos de encaminhamento, assim como as características sociodemográficas dessa população, os instrumentos psicométricos utilizados nas avaliaçôes e os níveis de diagnóstico dos usuários que receberam o diagnóstico.

\section{Método}

Este estudo utiliza como método a pesquisa documental retrospectiva, descritiva e exploratória de cunho quantitativo. Os dados foram coletados a partir dos prontuários de crianças com idade de zero a 12 anos incompletos, que iniciaram processo de avaliação no serviço, embora algumas não tenham concluído a triagem. Toda a amostra foi encaminhada com suspeita de diagnóstico de transtorno do espectro autista para o CER II de uma cidade do sul do Brasil.

O CER-II aqui estudado não atende outras deficiências intelectuais além do TEA, devido a uma pactuaçáo junto à gerência de saúde regional para que o serviço atendesse somente a população com suspeita de autismo, além da população com 
deficiências físicas. Por esta razão, os dados coletados foram dos prontuários dos pacientes com suspeita de TEA.

A região atendida pelo CER-II desta pesquisa é composta por 12 cidades em um raio de 90 quilômetros, com 689.912 habitantes no total, sendo que a população de 0 a 14 anos é de 124.692 (IBGE, 2010). No período de abril de 2014 a dezembro de 2017, 1.251 usuários utilizaram o serviço, incluindo os usuários com deficiência física e intelectual, 685 usuários especificamente com suspeita de TEA fizeram avaliação, na faixa etária de 0 a 12 anos. A coleta de dados se deu no período de maio a julho de 2018.

Foi elaborada pelos autores uma ficha para a sistematização dos dados de identificação da população foco do estudo: idade, sexo, escolaridade da criança, escolaridade dos pais, com quem reside, posição ocupada pela criança na prole, fonte de encaminhamento, profissão e idade dos pais, queixas desta população que motivaram a procura pelo serviço, resultados das escalas de rastreio para TEA utilizadas e se houve a conclusão do diagnóstico confirmando a suspeita de TEA. Para a análise dos dados coletados, foi realizada estatística descritiva, sendo esta constituída pelo conjunto de métodos destinados a organização, descrição e medidas de dados de uma populaçáo com o auxílio do programa SPSS versão 21.0.

A pesquisa foi aprovada pelo comitê de ética com seres humanos da universidade na data de 11 de abril de 2018, sob o número de parecer 2.592.249.

\section{Resultados}

Os dados do serviço mostram que em 2014, ano em que o serviço foi inaugurado, houve $19,1 \%$ ( $\mathrm{n}=131)$ de crianças realizando o processo de avaliação em relação a amostra total; o ano de 2015 teve 23,5\% ( $\mathrm{n}=161) ; 2016$ teve 30,7\% ( $\mathrm{n}=210)$; e 2017 teve $26,7 \%$ ( $n=183)$, conforme o gráfico a seguir. 
Gráfico 1. Crianças que iniciaram o processo de avaliação de acordo com o ano

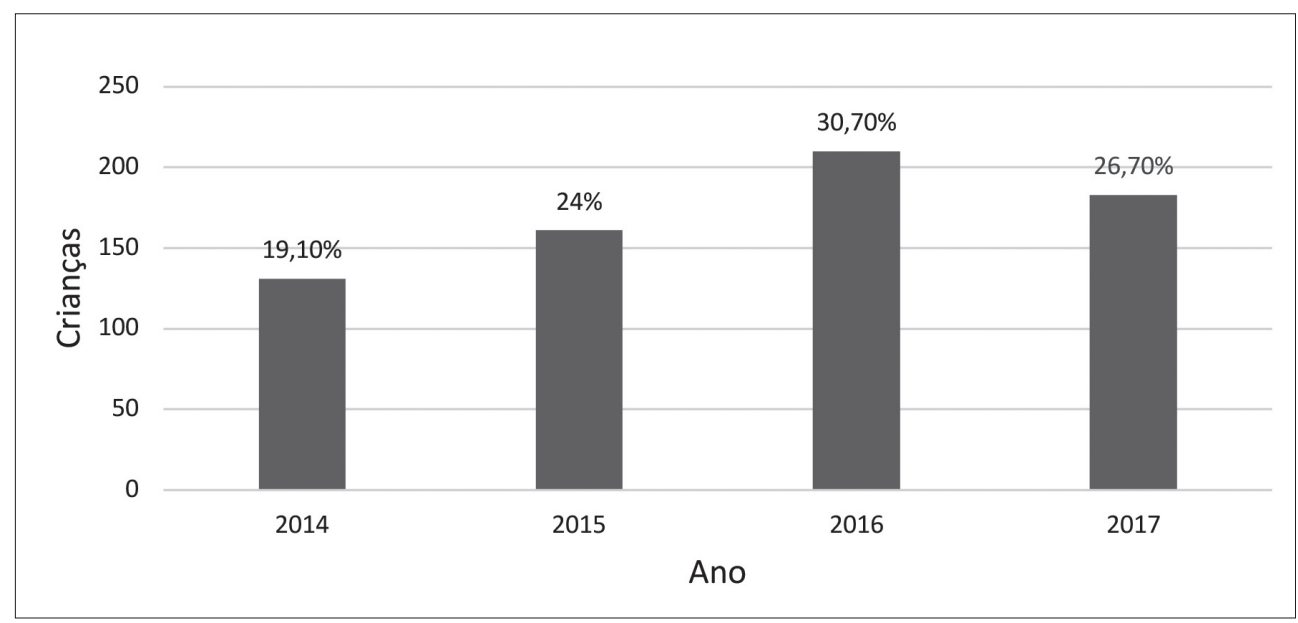

Fonte: elaboração própria.

Nos prontuários, consta que 10,5\% ( $\mathrm{n}=72)$ dos usuários foram desligadas a pedido dos pais; $55 \%(\mathrm{n}=380)$ receberam alta do serviço; $0,5 \%(\mathrm{n}=4)$ não continuou a avaliação por não preencher algum critério para continuar a mesma; 8,02\% $(\mathrm{n}=55)$ ainda estavam em processo de avaliação no serviço quando a coleta de dados aconteceu; $5,1 \%$ ( $n=35)$ já haviam recebido o diagnóstico de algum outro serviço e por esta razão não continuaram com o processo de avaliação e 20,3\% (n=139) das crianças atendidas foram desligadas do serviço por abandono, ou seja, o usuário faltou mais de duas vezes consecutivas sem apresentar uma justificativa ao serviço.

No que se refere ao sexo dos sujeitos, há maior frequência do sexo masculino, 83,8\% ( $\mathrm{n}=574$ ). A faixa de idade mais frequente encontra-se nos três anos, 17,2\% ( $\mathrm{n}=118)$; a média de idade é de 5,47 anos e a idade menos frequente é 12 anos, compondo $1,3 \%(\mathrm{n}=9)$ da amostra. A idade mínima é de até um ano, pois alguns usuários eram encaminhados com meses de idade, compondo 2,2\% ( $\mathrm{n}=15)$, e a idade máxima é 12 anos.

Em relação à escolaridade dos usuários, 42\% (n=288) estão na educação infantil; 27\% ( $n=185)$ encontram-se no ciclo de alfabetização, que consiste no primeiro, segundo e terceiro anos do ensino fundamental; 5,25\% (n=36) estáo no quarto ano do ensino fundamental; 4,23\% ( $\mathrm{n}=29)$ estão no quinto ano do ensino fundamental; $2,48 \%$ estão no sexto ano do ensino fundamental; $0,43 \%(\mathrm{n}=3)$ estão no sétimo ano 
do ensino fundamental. Dos $42 \%(\mathrm{n}=288)$ que se encontram na educação infantil, $40,2 \%$ ( $\mathrm{n}=276$ ) estão na faixa etária de zero a cinco anos - sendo assim, o nível de escolaridade condiz com a idade.

Apenas $0,14(\mathrm{n}=1)$ frequenta a APAE e o CER a mesmo tempo e $1,45 \%$ $(\mathrm{n}=10)$ não frequenta a escola. Entretanto, desses usuários que não frequentam a escola, $1,02 \%(n=7)$ tem de um a três anos e $0,43 \%(n=3)$, seis, nove e 11 anos. Em $0,87 \%(\mathrm{n}=6)$ dos prontuários constava a informação de que as crianças estavam frequentando a escola, mas não especificava o ano escolar. E em 16,05\% ( $\mathrm{n}=110)$ dos prontuários os profissionais do serviço não registraram informação referente à escolaridade do usuário.

Nos prontuários não havia informações sobre frequência em outras instituiçóes de educação especial, como a APAE, pois há um convênio entre a APAE e o SUS da região. Desta forma, as crianças que frequentam o CER não podem frequentar a APAE ou outras instituiçóes semelhantes que tenham convênio com o SUS.

Em relação aos dados sociodemográficos, analisou-se a composição familiar. $\mathrm{Na}$ primeira categoria, observou-se que 59,7\% ( $\mathrm{n}=409)$ das famílias seguem o modelo nuclear, composto por pai e mãe, criança e irmãos. Salienta-se que os padrastos e madrastas foram incluídos nesta categoria. A segunda composição familiar com maior frequência é a família ampliada, que é composta pela família nuclear juntamente com outros parentes como avós, tios, entre outros. Esse modelo tem frequência de 13\% (n=89) da amostra. A terceira composição familiar com maior frequência é o modelo monoparental, composta por apenas um dos genitores e o filho, sendo esta 10,8\% ( $\mathrm{n}=74)$ da amostra. Destas famílias monoparentais, 97,3\% $(\mathrm{n}=72)$ delas são chefiadas pelas mães. Ainda foram relatadas composições familiares em que as crianças residem com algum membro da família, como tia, madrinha, avó, sendo esta a composição familiar de 2,2\% (n=15) dos usuários. Já a composição familiar com a menor frequência $0,1 \%(n=1)$ é a família homoafetiva, que se trata de duas pessoas do mesmo sexo em relacionamento e residentes com um filho. Por fim, 14,2\% ( $\mathrm{n}=97)$ dos prontuários não continham a informação a respeito da composição familiar do usuário. A idade dos cuidadores variou de 19 até aos 70 anos, porém a maior concentração foi entre os 30 a 39 anos, sendo que os pais nessa faixa etária são $47,1 \%$ e as mães, $45,9 \%$.

Quanto à escolaridade dos cuidadores, esta teve variação entre não alfabetizado até o nível de pós-graduação. $\mathrm{O}$ resultado que predominou entre ambos foi ensino 
médio completo, sendo as mães $36,8 \%$ e os pais, $27,3 \%$. Logo, foram analisadas as profissōes de cada cuidador e percebeu-se que a maior frequência é a de mulheres que exercem a função de donas de casa (33,3\%). As outras duas ocupaçôes mais frequentes são de profissóes relacionadas a limpeza $(10,8 \%)$ e profissóes ligadas a áreas da administração e finanças (9,9\%). Entre os homens, 24,4\% trabalham em áreas de construção e manutenção e $12,4 \%$ trabalham em serviços operacionais.

$\mathrm{Na}$ tabela 1, são apresentados os sinais identificados que motivaram o encaminhamento para o serviço. Esses foram coletados através do protocolo de encaminhamento realizado pelos profissionais da atenção primária para o CER. Vale ressaltar que varia a quantidade de sinais relatados nos encaminhamentos para cada usuário. Dentre os possíveis sinais categorizados que estão relacionados aos critérios do diagnóstico de TEA, conforme o DSM-5, encontram-se os comportamentos estereotipados e/ou interesses restritos, a hipersensibilidade, além dos déficits na linguagem. Este último é o sinal que aparece com maior frequência, em 58,8\% $(\mathrm{n}=402)$ dos sujeitos.

Quantos aos possíveis sinais associados ao TEA, destacam-se os déficits cognitivos, sinais relacionados à atenção ou concentração, além dos comportamentos internalizantes e os externalizantes. Nestes dois últimos foram categorizdos, no primeiro caso, os comportamentos referentes a sentimentos de medo, inferioridade, depressão, retraimento e ansiedade. No segundo, classificaram-se aqueles referentes à dificuldade no controle de impulsos, hiperatividade, agressôes ou qualquer outro tipo de conflito ambiental. Já no déficit cognitivo foram categorizados sinais relacionados a dificuldades de aprendizagem e/ou inteligência. As categorias mais recorrentes são os comportamentos internalizantes, com 49,5\% ( $\mathrm{n}=339)$, e os comportamentos externalizantes, com 56,9\% ( $\mathrm{n}=390)$.

Também foram identificados alguns comportamentos que motivaram o encaminhamento para o serviço, mas que não possuem relação direta com o diagnóstico de TEA. Estes foram classificados como controle dos esfíncteres, dificuldades no sono e sinais físicos, como dificuldades psicomotoras. 
Tabela 1. Sinais referentes ao diagnóstico de TEA

\begin{tabular}{lcc}
\hline Variáveis & $\mathrm{n}=685$ & $\%$ \\
\hline Sinais & & \\
Déficits na linguagem & 402 & $58,8 \%$ \\
Comportamentos externalizantes & 390 & $56,9 \%$ \\
Comportamentos internalizantes & 339 & $49,5 \%$ \\
Déficit cognitivo & 157 & $22,9 \%$ \\
Sinais relativos à atenção/concentração & 134 & $19,6 \%$ \\
Comportamentos estereotipados e/ou interesses restritos & 117 & $17,1 \%$ \\
Sinais físicos & 43 & $6,3 \%$ \\
Hipersensibilidade & 40 & $5,8 \%$ \\
Dificuldades no sono & 38 & $5,5 \%$ \\
Controle dos esfíncteres & 27 & $3,9 \%$ \\
\hline
\end{tabular}

Fonte: elaboração própria.

Posteriormente, buscaram-se informações referentes ao processo de diagnóstico de TEA dos usuários do serviço. Evidencia-se que das 685 crianças que iniciaram o processo de avaliação, $206(30,1 \%)$ receberam o diagnóstico de TEA, das quais 135 informaram algum nível de gravidade de TEA que foram categorizados em leve, leve-moderado, moderado, moderado-grave e grave. Dentre essas categorias, as mais frequentes foram a leve, em 61 usuários, e a moderada, com 57.

Outros $200(29,2 \%)$ usuários não preenchiam os critérios de diagnóstico de autismo. Destes, 50 informaram a existência de outro diagnóstico em vez de TEA, sendo os mais frequentes os transtornos de déficit de atenção e hiperatividade, em 16 casos e algum transtorno da fala e da linguagem, em 13. É importante ressaltar que o CER não trabalha com diagnóstico diferencial de outros transtornos mentais, o que justifica a baixa frequência de outros diagnósticos que não sejam o de TEA. Os demais usuários, compondo o restante dos 40,7\% ( $\mathrm{n}=279)$, não receberam um diagnóstico do serviço, porque nessa amostra encontram-se alguns dos usuários que haviam sido desligados por abandono, a pedido dos pais, já haviam sido diagnosticados por outros serviços ou ainda estavam em processo de avaliação no CER. 
O diagnóstico realizado pelos profissionais é feito baseado no CID-10. Entretanto, para conseguir definir o nível do TEA, os profissionais realizam uma transposição para o DSM-5. O mesmo é concluído pela equipe multiprofissional, juntamente com o médico neurologista.

Para uma melhor compreensão da formulação desse processo, buscou-se identificar os instrumentos utilizados no processo de diagnóstico de TEA no serviço. Pode-se observar que todos os cinco instrumentos utilizados são para o rastreamento de sinais e sintomas de TEA, e não necessariamente para o diagnóstico: Autism Behavior Checklist (ABC), uma lista de checagem com 57 comportamentos atípicos; Escala de Avaliação de Traços Autísticos (ATA); Childhood Autism Rating Scale (CARS), que é uma entrevista semiestruturada; Modified Checklist for Autism in Toddlers (M-CHAT), para rastreio de sinais precoces, e a Escala Vineland de Comportamento Adaptativo, que faz a análise das atividades de vida diária (AVDs) e comunicação (BOSA; TEIXEIRA, 2017). Destas, a mais utilizada foi a $A B C$, sendo usada em 70,5\% ( $n=483)$ dos usuários. A segunda mais utilizada foi a ATA, com 65,7\% (n=450); a terceira foi a Vineland, 64,7\% ( $n=443)$; a quarta foi a escala CARS, com $56,9 \%(\mathrm{n}=390)$; e a menos utilizada foi a M-CHAT, com $15,3 \%(\mathrm{n}=105)$.

É importante ressaltar que os profissionais costumam utilizar mais de uma escala nas avaliações e que essa escolha se dá a partir da idade da criança e de protocolos internos do serviço.

Tabela 2. Níveis de cada escala

\begin{tabular}{lcc}
\hline Variáveis & $\mathrm{N}$ & $\%$ \\
\hline Nível M-CHAT $(\mathrm{n}=105)$ & & \\
Com TEA & 68 & $64,8 \%$ \\
Sem TEA & 37 & $35,2 \%$ \\
Nível ABC $(\mathrm{n}=483)$ & & \\
Autismo & 211 & $43,7 \%$ \\
Provável autismo & 100 & $20,7 \%$ \\
Sem autismo & 172 & $35,6 \%$
\end{tabular}




\begin{tabular}{lcc}
\hline Variáveis & $\mathrm{N}$ & $\%$ \\
\hline Nível CARS (n=390) & 223 & $57,2 \%$ \\
Sem TEA & 104 & $26,7 \%$ \\
Leve/Moderado & 63 & $16,2 \%$ \\
Grave & & \\
Nível ATA (n=450) & 244 & $54,2 \%$ \\
Com autismo & 206 & $45,8 \%$ \\
Sem autismo & & \\
Nível VINELAND (n=443) & 197 & $44,5 \%$ \\
Suave/leve & 157 & $35,4 \%$ \\
Moderado & 29 & $6,5 \%$ \\
Grave & 19 & $4,3 \%$ \\
Profundo & 30 & $6,8 \%$ \\
Adequado & 11 & $2,5 \%$ \\
Limítrofe & & \\
\hline
\end{tabular}

Fonte: elaboração própria.

Quanto aos níveis das escalas, estes foram categorizados conforme os níveis preestabelecidos na padronização de cada instrumento. No caso do M-CHAT, se haveria ou não indícios de TEA, sendo que de 105 aplicaçóes, 64,8\% indicariam o transtorno. Quanto aos níveis do $\mathrm{ABC}$, a classificação dá-se em autismo, provável autismo e sem autismo. O primeiro, com 43,7\% de 483 usuários, é o mais frequente. O ATA avalia também a presença ou não de autismo. Observa-se um equilíbrio quanto aos resultados, ainda que maior para o diagnóstico de autismo, sendo que, de 450 prontuários, 54,2\% indicam TEA. No que concerne ao CARS, é um dos testes utilizados para a descrição do nível de autismo, indicando a inexistência de autismo, se possui, em grau leve/moderado ou se possui em grau grave. Observa-se que a maior parte dos resultados referentes aos 390 usuários que utilizaram esse instrumento $(57,2 \%)$ não indica TEA. Por fim, outro instrumento utilizado foi o Vineland, que analisa o comportamento adaptativo do sujeito. Nele nota-se uma diversidade maior quanto às categorias desde adequada, limítrofe, leve, moderada, grave e profunda. 
Evidencia-se que as categorias mais frequentes nos 443 prontuários que informam o uso desse instrumento são a leve, com $44,5 \%$, e a moderada, com $35,4 \%$.

\section{Discussão}

Criada em 2012, a Rede de Cuidados à Pessoa com Deficiência tinha em um dos seus principais objetivos a implantação dos Centros Especializados em Reabilitação em todo o Brasil nos anos seguintes (BRASIL, 2013). Em 2014, foi implantado o serviço da presente pesquisa, justificando assim a menor frequência de usuários no ano de 2014, com uma porcentagem de 19,1\% (n=131) em relação à amostra total.

No que se refere às questóes relativas ao sexo, a procura pelo serviço por usuários do sexo masculino foi bastante prevalente, compondo de forma significativa a amostra. De acordo com o DSM-5, o transtorno do espectro autista é quatro vezes mais frequente no sexo masculino (APA, 2014). Esse dado também é reforçado por um estudo do Centers for Disease Control and Prevention, realizada nos Estados Unidos, que mostra a prevalência do autismo no sexo masculino comuma razão de 4:1 (CHRISTENSEN et al., 2016).

A avaliação precoce tem se mostrado importante, levando em consideração que quanto antes o diagnóstico, melhor o prognóstico das crianças com TEA. Os dados mostram que a procura pelo serviço dos usuários que se encontram nos quatro primeiros anos de idade foi predominante, compondo $45,6 \%(n=313)$ da população. Uma avaliação e diagnóstico precoce é importante tanto para melhor intervenção e manejo dos sintomas, mas também no que tange ao fortalecimento da rede de apoio, que é composta pela família. Além disso, uma intervenção precoce auxilia em um melhor desempenho acadêmico por parte das crianças que possuem TEA (ELDEVIK et al., 2009).

Sobre a escolaridade das crianças, é válido citar que $42 \%(\mathrm{n}=288)$ da amostra deste estudo estão inseridas na educação infantil regular; destas, 276 crianças estão na faixa etária de um a cinco anos, condizendo desta maneira a idade com a escolaridade. Dos 40,2\% que estão inseridas no ensino fundamental, todas se encontram na faixa etária de cinco a 12 anos. É importante destacar, que a Lei no 12.764, de 27 de dezembro de 2012, instituiu a Política Nacional de Proteção dos Direitos da Pessoa com Transtorno do Espectro Autista, reforçando a ideia constituinte do direito à educação (BRASIL, 2012b). 
Os dados relativos à família dos usuários também aparecem com destaque, visto que é o contexto inicial em que as primeiras relaçôes desses sujeitos são estabelecidas. De acordo com os dados identificados no serviço, a maior parte das famílias configura-se como nuclear. Esse resultado conversa com os resultados obtidos pelo Instituto Brasileiro de Geografia e Estatística (IBGE), em 2012, quando realizou estudo a respeito das configuraçóes de todas as famílias com base nos resultados do Censo de 2010. Desses dados, conclui-se que das 49.975.934 famílias investigadas em todo o Brasil, 49\% seriam famílias de casais com filhos, ou seja, do tipo nuclear, sendo o mais recorrente em território nacional (IBGE, 2012). Outro dado relevante é que quase todas as famílias monoparentais se constituem tendo a mãe como cuidadora principal. A literatura coloca as mães como os sujeitos que mais se afetam com sobrecarga emocional, física e financeira no cuidado das crianças com autismo, aumentando o risco de desenvolver estresse, depressão e ansiedade (CHRISTMANN et al., 2017).

No que concerne aos dados dos pais nos últimos anos, tem-se discutido a idade dos genitores como possível fator de riscos. Um estudo de caso-controle realizado por Maia et al. (2018) mostra associação entre TEA e idade avançada dos pais quando ambos os genitores possuem idades avançadas. Conforme citado nos resultados, a maioria das idades desses cuidadores no momento da realização do diagnóstico pelo serviço, tanto para mães, como para pais, encontra-se na faixa dos 30-39 anos. Hipotetiza-se, devido à maioria das idades das crianças no momento de diagnóstico estar na faixa de $2-4$ anos (43,4\% da amostra), que conforme os estudos vêm indicando, haver relação entre idade avançada e o diagnóstico de TEA, pois a maior parte dos pais do estudo tiveram seus filhos a partir dos 35 anos de idade. Fazse necessário ressaltar a importância da realização de acompanhamento de crianças geradas por pais em idades avançadas, por conta de esse fator estar associado com o diagnóstico de TEA (MAIA et al., 2018).

Enfatiza-se nessa pesquisa o SUS enquanto política pública de acesso à saúde para diferentes classes socioeconômicas. Já que os dados mostram que há maior prevalência de usuários que possuem cuidadores com ensino médio completo e que possuem ocupaçôes que não exigem nível superior de ensino, diferentemente do contexto norte-americano relatado por outra pesquisa, que mostra que $72 \%$ das mães de crianças autistas possuem nível educacional acima do ensino médio completo (LIPTAK et al., 2008). Apesar disso, a utilização do serviço também pode 
ser controversa, podendo indicar privilégio no acesso ao CER, promovendo assim um processo de exclusão dos usuários de famílias com menor escolaridade e maior vulnerabilidade, já que $53,9 \%$ da população brasileira com mais de 25 anos possuem nível de escolaridade até o ensino médio incompleto, variando de sem instrução até o ensino médio incompleto (IBGE, 2017).

Quanto aos sinais e sintomas das crianças com espectro autista, de acordo com a literatura, os primeiros sinais começam a ser percebidos pelos pais entre os $12 \mathrm{e}$ 24 meses, fazendo-se presente de forma mais significativa os déficits relacionados à atenção, os déficits relacionados à linguagem e os comportamentos externalizantes, como agressividade e hiperatividade (ZAQUEU et al., 2015). Outros indicadores que se fazem presentes mas com início em uma idade diferenciada, que seria a partir dos 18 meses, são os sinais de hipersensibilidade e comportamentos estereotipados (ZWAIGENBAUM et al., 2009).

Em relação aos sinais que motivaram o encaminhamento da população desta pesquisa para o CER, o mais prevalente foram os déficits na linguagem, em 59,8\% da amostra. $O$ déficit na linguagem condiz com nenhuma ou pouca aquisição na fala, assim como ecolalia. O segundo sinal que mais motivou o encaminhamento foram os comportamentos externalizantes; e o terceiro foram os comportamentos internalizantes. Os encaminhamentos realizados pela rede de atenção primária para o serviço por conta do déficit de linguagem, do comportamento externalizante, dos déficits de atenção, hipersensibilidade e comportamentos estereotipados condizem com os dados da literatura no que diz respeito aos sinais precoces (BOSA; TEIXEIRA, 2017). No que concerne aos comportamentos internalizantes, os mesmos não são considerados sinais precoces pela literatura (BOSA; TEIXEIRA, 2017) e motivaram 49,5\% ( $\mathrm{n}=339)$ dos encaminhamentos.

É necessário realizar uma boa triagem na atenção primária, para motivar o encaminhamento para o CER. Pesquisas mostram que muitas vezes os cuidadores percebem os sinais e sintomas, mas ao relatarem para o médico, os mesmos não associam os sintomas com o TEA (OZONOFF; GOODLIN-JONES; SOLOMON, 2005).

Em relação à população atendida no CER nesta pesquisa, é importante ressaltar que a amostra de 685 usuários é de crianças que residem em 12 cidades. Essas cidades possuem uma população de 124.692 habitantes que se encontram na faixa etária de 0 a 14 anos. Dos 685 encaminhamentos, 206 (30,1\%) tiveram a 
confirmação do diagnóstico de TEA. Destaca-se aqui um baixo número de pesquisas epidemiológicas sobre o assunto no Brasil. Em pesquisas de recorte clínico esses dados são inexistentes, dificultando uma discussão a respeito do fato (GOMES et al, 2015). Deve-se considerar que a implementação do serviço é recente e que existe a possibilidade do uso de outros serviços de saúde, como a rede privada ou instituiçôes filantrópicas que atendem essas demandas, como as APAEs.

Dos cinco instrumentos utilizados pelo serviço no rastreamento de TEA, a M-CHAT é a única recomendada pelo Ministério da Saúde (BRASIL, 2014). Entretanto, segundo revisão sistemática realizada por Seize e Borsa (2017), em que se objetivou identificar instrumentos disponíveis para rastreamento dos sinais de TEA, não há consenso em relação às propriedades psicométricas da M-CHAT. Em vista disso, entende-se que alguns estudos representam um avanço, mas que isto não é o bastante para considerar a escala validada e adequada para uso livre segundo recomenda o Ministério da Saúde (SEIZE; BORSA, 2017).

O uso da M-CHAT também entra em debate após a Lei no 13.438 de 2017, que preconiza o emprego de protocolo com parâmetros para a avaliação de riscos para desenvolvimento psíquico das crianças, pelo SUS. Assim, o Ministério da Saúde apresentou uma nova recomendação, constatando que a Caderneta de Saúde da criança é o instrumento por excelência de vigilância e rastreamento de atrasos do desenvolvimento, sendo um "instrumento de vigilância integral à saúde" (BRASIL, 2008; 2017).

Nesse aspecto, também se salienta a importância de instrumentos que possibilitem avaliar os níveis de funcionalidade, para que possam ser adaptadas as intervençôes necessárias para cada caso. O DSM-5 coloca que esses níveis de funcionalidade dizem respeito aos interesses restritos/comportamentos repetitivos e a comunicação social, podendo ser no nível 1 (exigindo apoio), nível 2 (exigindo apoio substancial) e no nível 3 (exigindo apoio muito substancial) (APA, 2014). No caso do serviço em questão, como já citado, o nível de funcionalidade foi categorizado entre leve, moderado e grave, entendendo-se assim que esses níveis se equivalem aos do DSM5. Sendo assim, permitiu-se avaliar que 135 dos 206 usuários com diagnóstico de TEA tiveram seus níveis de funcionalidade avaliados, sendo os mais comuns o leve e o moderado. Na literatura, não foi possível identificar algum estudo que colocasse qual grau de funcionamento seria o mais frequente, apenas a ocorrência ou não 
do transtorno e seu QI, sendo que este não se fez presente em casos dos níveis de funcionalidade 1 ou leve (APA, 2014; FOMBONNE, 2009).

\section{Conclusão}

Este estudo permitiu concluir que os principais motivos de encaminhamento para o serviço estão relacionados a déficits na linguagem e a problemas de comportamento externalizantes e internalizantes. Também possibilitou descrever suas características sociodemográficas, constatando que a maior parte dos usuários faz parte de uma família nuclear. Identificou que aproximadamente um terço das avaliaçóes realizadas denota autismo, sendo que em 65,63\% desses diagnósticos houve especificação do nível, denotando uma maioria em leve ou moderada, e que o instrumento mais utilizado pelos profissionais foi o $\mathrm{ABC}$. Vale ressaltar que houve o predomínio significativo do sexo masculino.

As políticas públicas de saúde no Brasil voltadas para essa população são recentes, e que os CER foram criados há menos de dez anos. A unidade aqui investigada funciona há cerca de quatro anos, sendo assim um serviço com pouco tempo de aplicação. Destaca-se a necessidade de profissionais e instrumentos qualificados no serviço, tanto para o processo de diagnóstico quanto de intervenção.

Como toda política pública implementada, ela deve ser avaliada mediante seus preceitos e objetivos predeterminados, verificando-se sua efetividade. No serviço referido, objetiva-se promover cuidados em saúde no processo de reabilitação física e intelectual, assim como identificar precocemente os déficits e desenvolver meios para a promoção da saúde e prevenção de agravos dos usuários. Levando em conta apenas os dados identificados para TEA, esta pesquisa evidenciou efetividade no processo de diagnóstico precoce dos usuários. Ainda assim, devido aos objetivos desta pesquisa, não foi possível analisar as práticas de reabilitação posteriores ao diagnóstico, e chama a atenção a quantidade de prontuários com informaçóes incompletas.

Por fim, para a realização de novas pesquisas, sugere-se a avaliação das intervençôes realizadas pelo serviço e seus profissionais, e também como se dá a ocorrência da relação do serviço com a Atenção Primária à Saúde. Além disso, propóe-se a realização de outros estudos epidemiológicos sobre TEA no país, para maior generalização e clareza dos dados obtidos em demais contextos, especialmente no que tange à verificação dos níveis de funcionalidade mais recorrentes. ${ }^{1}$ 


\section{Agradecimentos}

Os autores agradecem o subsídio financeiro do Governo do Estado de Santa Catarina através da bolsa pesquisa do Artigo 170, assim como à equipe do CER.

\section{Referências}

ASSOCIACÃO AMERICANA DE PSICOLOGIA (APA). Manual diagnóstico e estatístico de transtornos mentais: DSM-5- 5. ed. Porto Alegre-RS: Artmed, 2014.

BACKES, B. et al. Psychometric properties of assessment instruments for autism spectrum disorder: a systematic review of Brazilian studies. Jornal Brasileiro de Psiquiatria, v. 63, n. 2, p. 154-164, 2014.

BOSA, C. A.; TEIXEIRA, M. C. T. V. (Org.). Autismo: avaliação psicológica e neuropsicológica. São Paulo: Hogrefe, 2017.

BRASIL. Ministério da Saúde. Caderneta de saúde da criança: Passaporte da cidadania. 5. ed. Brasília: Ministério da Saúde, 2008. 84 p.

. Ministério da Saúde. Centros Especializados em Reabilitação - CER. 2017. Disponível em: <http://portalms.saude.gov.br/artigos/808-pessoa-com-deficiencia/41078-centros-especializadosem-reabilitacao-cer>. Acesso em: 09 set. 2018.

. Ministério da Saúde. Secretaria de Direitos Humanos. Avanço das políticas públicas para as pessoas com deficiência: uma análise a partir das Conferências Nacionais. Brasília: MS, 2012a.

. Ministério de Saúde. Manual de utilização da Caderneta de Saúde da Criança. Brasília: Ministério da Saúde, 2005. 22 p.

- Ministério de Saúde. Secretaria de Atenção à Saúde. Departamento de Ações Programáticas Estratégicas. Diretrizes de Atenção à Reabilitação da Pessoa com Transtornos do Espectro do Autismo (TEA). Brasília: Ministério da Saúde, 2014. 88 p.

. Presidência da República. Decreto n 6949, de 25 de agosto de 2009. Promulga a Convenção Internacional sobre os Direitos das Pessoas com Deficiência e seu Protocolo Facultativo, assinados em Nova York, em 30 de março de 2007. Disponível em: <http://www. planalto.gov.br/ccivil_03/_ato2007-2010/2009/decreto/d6949.htm>. Acesso em: 05 abr. 2019.

. Presidência da República. Lei no 12.764, de 27 de dezembro de 2012. Política Nacional de Proteção dos Direitos da Pessoa com transtorno do espectro autista, 2012b. Disponível em: <http:// www.planalto.gov.br/ccivil_03/_ato2011-2014/2012/lei/112764.htm>. Acesso em: 27 jun. 2017.

. Presidência da República. Lei n 13.146 , de 6 de julho de 2015. Aprova a lei brasileira de inclusão da pessoa com deficiência (Estatuto da Pessoa com Deficiência), 2015. Disponível em: http://www.planalto.gov.br/ccivil_03/_Ato2015-2018/2015/Lei/L13146.htm. Acesso em: 05 abr. 2019. 
. Presidência da República. Lei no 13.438, de 26 de abril de 2017. Torna obrigatória a adoção, pelo Sistema Único de Saúde (Sus), de protocolo que estabeleça padrôes para a avaliação de riscos para o desenvolvimento psíquico das crianças. Brasília, DF, 2017. Disponível em: <http:// www.planalto.gov.br/ccivil_03/_Ato2015-2018/2017/Lei/L13438.htm>. Acesso em: 08 abr. 2019.

. Presidência da República. Secretaria de Direitos Humanos. Plano Nacional dos Direitos da Pessoa com Deficiência - Viver sem Limites. Brasília: Secretaria Nacional de Promoção dos Direitos da Pessoa com Deficiência, 2013. 92 p.

CAMPOS, M. F.; SOUZA, L. A. P.; MENDES, V. L. F. A rede de cuidados do Sistema Único de Saúde à saúde das pessoas com deficiência. Interface: comunicação, saúde, educação, v. 19, n. 52, p. 207-2010, 2015.

CHRISTENSEN, D. L. et al. Prevalence and Characteristics of Autism Spectrum Disorder Among Children Aged 8 Years-Autism and Developmental Disabilities Monitoring Network. Morbidity And Mortality Weekly Report: Surveillance Summaries, United States, v. 6, n. 67, p.123, 2016.

CHRISTMANN, M. et al. Estresse Materno e necessidade de cuidado dos filhos com TEA na perspectiva das mães. Cadernos de Pós-graduação em Distúrbios do Desenvolvimento, São Paulo, v. 17, n. 2 , p. $8-17,2017$.

ELDEVIK, S. et al. Meta-analysis of early intensive behavioral intervention for children with autism. Journal OfClinical Child \& Adolescent Psychology, London, v. 3, n. 38, p.439-450, 2009. FOMBONNE, E. Epidemiology of pervasive developmental disorders. Pediatric Research, n. 65, v. 6, p. 591-598, 2009.

GOMES, P. T. M. et al. Autismo no Brasil, desafios familiares e estratégias de superação: revisão sistemática. Revista de Pediatria, Rio de Janeiro, v. 91, n. 5, p. 111-121, 2015.

HILL, A. P.; ZUCKERMAN, K.; FOMBONNE, E. Epidemiology of autism spectrum disorders. In: VOLKMAR et al. (Org.). Handbook of Autism and Pervasive Developmental Disorders. V. 1. Diagnosis, Development and Brain Mechanisms. Wiley, 2014, p. 57-96.

INSTITUTO BRASILEIRO DE GEOGRAFIA E ESTATÍSTICA. Análise do censo populacional de 2010. Rio de Janeiro: IBGE, 2010. Disponível em: <https://ww2.ibge.gov.br/ home/estatistica/populacao/censo2010/default.shtm>. Acesso em: 2 out. 2018.

. Censo demográfico de 2010: famílias e domicílios Resultados da amostra. Rio de Janeiro: Ministério do Planejamento, Orçamento e Gestão, 2012.

. Pesquisa Nacional por Amostra de Domicílios Contínua. Censo Escolar. PNADC 2017.

Rio de Janeiro: IBGE, 2017. Disponível em: https://biblioteca.ibge.gov.br/visualizacao/livros/ liv101576_informativo.pdf. Acesso em: 15 abr. 2019. 
LIPTAK, G. S. et al. Disparities in Diagnosis and Access to Health Services for Children with Autism: Data from the National Survey of Children's Health. Journal of Developmental \& Behavioral Pediatrics, [s.1.], v. 29, n. 3, p. 152-160, jun. 2008.

MAIA, F. A. et al. Transtorno do espectro do autismo e idade dos genitores: estudo de casocontrole no Brasil. Cad. Saúde Pública. Rio de Janeiro, v. 34, n. 8, p. e00109917, 2018.

MAZZOTTA, M. J. S. Educação Especial no Brasil. História e Políticas Públicas. 2.ed. São Paulo: Cortez, 1999.

OLIVEIRA, B. D. C. et al. Políticas para o autismo no Brasil: entre a atenção psicossocial e a reabilitação. Physis: Revista de Saúde Coletiva. Rio de Janeiro, v. 27, n. 3, p. 707-726, 2017.

OZONOFF, S.; GOODLIN-JONES, B. L.; SOLOMON, M. Evidence-based assesment of Autim Spectrum Disorders in children and adolescents. Journal of Clinical Child \& Adolescent Psychology, v. 34, p. 523-540, 2005.

PAULA, C. S. et al. Autism in Brazil - perspectives from science and society. Revista da Associação Médica Brasileira, São Paulo, v. 57, p. 2-5, 2011a.

Prevalence of pervasive developmental disorder in Brazil: a pilot study. Journal of Autism and Developmental Disorders. São Paulo, v. 41, p. 1738-1742, 2011 b.

PORTOLESE, J. et al. Mapeamento dos serviços que prestam atendimento a pessoas com transtorno do espectro autista no Brasil. Cad. Pós-Grad. Distúrb. Desenvolv. São Paulo, v. 17, n. 2, p. 79-91, dez. 2017.

PRESMANES, A. et al. Epidemiology of autism spectrum disorders. In: VOLKMAR et al. (Org.). Handbook of Autism and Pervasive Developmental Disorders. V. 1. Diagnosis, Development and Brain Mechanisms, Wiley, 2014, p. 57-96.

SEIZE, M. M.; BORSA, J. C. Instrumentos para Rastreamento de Sinais Precoces do Autismo: Revisão Sistemática. Psico-USF. Itatiba, v. 22, n. 1, p. 161-176, 2017.

ZAQUEU, L. C. C. et al. Associaçóes entre sinais precoces de autismo, atenção compartilhada e atrasos no desenvolvimento infantil. Psicologia: Teoria e Pesquisa, v. 31, n. 3, p. 293-302, 2015.

ZWAIGENBAUM, L. et al. Clinical assessment and management of toddlers with suspected Autism Spectrum Disorder: insights from studies of high-risk infants. Pediatrics, v. 123, n. 5 , p. 1381-1391, 2009.

\section{Nota}

${ }^{1}$ C.C. Rocha realizou coleta e tabulação de dados, levantamento bibliográfico e redação do artigo. S. M. Velasques realizou coleta de dados, levantamento bibliográfico e redação do artigo. A. F. Costa realizou tabulação de dados, levantamento bibliográfico e redaçáo do artigo. J. R. M. Portes orientou o levantamento bibliográfico e orientou a elaboração do artigo. 


\section{Abstract}

\section{The profile of the child population with suspected diagnosis of autism spectrum disorder attended by a Specialized Rehabilitation Center in a southern Brazilian city} The CER-II Specialized Rehabilitation Center is a SUS service that works interdisciplinarly in the assessment and rehabilitation of people with disabilities, including the population with autism. This research aimed to characterize the profile of children with suspected autistic spectrum disorder treated by a CER-II from southern Brazil, to survey sources, referral reasons, socio-demographic characteristics, psychometric instruments used in the assessments and the levels of diagnosis received. This retrospective, descriptive and exploratory-quantitative documentary study assessed 685 medical records of users aged 0 to 12 years old who were evaluated between 2014 and December 2017. The results showed that the average age of the sample is 5.47 years; $83.8 \%$ are male, $42 \%$ are in kindergarten and $59.7 \%$ are part of a nuclear family. The signs that most motivated referrals were language deficits (58.8\%) and externalizing behaviors (56.9\%); $30.1 \%$ of the sample had a confirmed ASD diagnosis and the most used instrument was the $\mathrm{ABC}(70.5 \%)$. It is observed the effectiveness of the application of the service proposed by this public policy, highlighting the need for qualified instruments for diagnostic evaluation and continuing training for professionals who work in it.

Keywords: autism; children; SUS. 